\title{
Relevant Influence of Semantic Web Framework on Smart E-Learning Environment
}

\author{
https://doi.org/10.3991/ijet.v16i17.24105
}

Subhabrata Sengupta $\left({ }^{\varpi}\right)$, Anish Banerjee, Satyajit Chakrabarti

Institute of Engineering and management, Kolkata, India

subhabrata.sengupta@iemcal.com

\begin{abstract}
E-Learning systems have unbound prospects to deliver unmatched effective learning services and feedback assistance than what it is presently offering through mediums like online tutoring, or other electronic educational management services. Different stages and application potentials of Semantic Web technology and its architecture can be applied at different sectors and phases of the E-Learning framework to amplify the quality and versatility of services. Features of Semantic Web have been explored in the sectors with respect to instructors to plan, analyse and execute their tasks and also in making a sustainable system that interprets the architecture of self-organized, distributed, and self-instructed online education to monitor it's influence on performance. The primary aim of this experiment is to learn how electronic and online learning frameworks can be improved and enhanced by the influence of semantic web technologies in understanding and simplifying concept clarification and description, reusable learning objects (LOs), and benefits of the applying ontology in describing the learning materials for a better and more efficient learning system.
\end{abstract}

Keywords-E-learning, semantic web, learning objects, ontology, efficient learning system.

\section{Overview}

Influence of online education and learning is growing in all spaces and areas of education, especially with the exponential increase of audience and prospects associated with it. The main positives associated with E-Learning, which is attracting a global audience towards it in huge numbers is its versatility in presenting education to its audience, decentralize learning making it accessible to anybody at any place of the world, maintaining consistent quality of learning and evaluation, personal grooming and individualized learning to suite and tune according to personal preference in terms of pace, adaptability, location, stretch of time, rescheduling and adaptation style of education. Although, a modern and efficient solution of online learning should meet the expanding requirements of the learners and educators. Smart information retrieval based on accurate query analysis and provides an interactive experience. 
The world wide web is a powerful tool for exploring but is bottle-necked by the fact that users have to navigate through the global content where efficiency is reduced due too presence of vague content as well. Semantic Web features are a way out of this problem, by promoting a new world wide web architecture that not only supports online material, and also formal semantics associated with it [1]. Semantic Web is a flourishing piece of technology as it provides an interactive environment between the learner or educator and the machine with which it is working with. The two main principles or residing blocks of Semantic Web are familiar formats for data interchange unlike the interchange of documents over the internet. Also, the importance of language for data recording which allows this man and machine to navigate through conceptually interlinked databases.

This paper is a structured explanation of the advanced semantic web architecture, it's influence in improving the prevailing E-Learning system and what issues can be solved by which feature of semantic web.

\section{$2 \quad$ E-Learning system characteristics}

The need of the hour, prospects of E-learning have been very appealing to the extent that it has seen an exponential growth in audience and users. Boosting the traditional training procedure and enhancing the prevailing system with more consistency, globalization and adaptable. It is trying to overcome the shortcomings of classical training and learning systems like: lack of personalization, linear and static way of learning. An ideal learning system must be fast, accurate, relevant - achieved by a distributed learner oriented, customizable dynamic(non-linear) form of learning as provided by E-Learning [2]. Main characteristics presented by the E-learning system include:

- Learner oriented study scheduling

- Interactive learning and software communication and navigation

- Non Linear content access

- Systematic curriculum

- Continuous learning modules

- Distributed content with sub divisions for repeated concept clearing, reference and a modular approach

- Personalization in learning and adaptive scheduling studies

- Dynamic content availability

Table 1. Differences between E-Learning and Traditional Training/Learning

\begin{tabular}{|l|l|l|}
\hline Point of Difference & \multicolumn{1}{|c|}{ E-Learning } & \multicolumn{1}{|c|}{ Traditional Learning/Training } \\
\hline Delivery type & Student oriented and driven - Pull & Instructor driven - Push \\
\hline Accessibility & $\begin{array}{l}\text { Non Linear Dynamic access to any part of } \\
\text { the curriculum at any given time or situa- } \\
\text { tion }\end{array}$ & $\begin{array}{l}\text { Linear or static flow of curriculum } \\
\text { with no random access to random } \\
\text { parts }\end{array}$ \\
\hline Responsiveness & $\begin{array}{l}\text { Reaction type - responding instantaneously } \\
\text { to the situation at hand }\end{array}$ & $\begin{array}{l}\text { Anticipatory type - pretends to antici- } \\
\text { pate the problem from before }\end{array}$ \\
\hline
\end{tabular}


Paper-Relevant Influence of Semantic Web Framework on Smart E-Learning Environment

\begin{tabular}{|l|l|l|}
\hline Point of Difference & \multicolumn{1}{|c|}{ E-Learning } & \multicolumn{1}{|c|}{ Traditional Learning/Training } \\
\hline Modality & $\begin{array}{l}\text { Continuous learning in loops without } \\
\text { breaking the flow }\end{array}$ & $\begin{array}{l}\text { Discrete training in selected chunks } \\
\text { with defined end points }\end{array}$ \\
\hline Authority & $\begin{array}{l}\text { Distributed authority where Content comes } \\
\text { from the participants- educator instruction }\end{array}$ & $\begin{array}{l}\text { Centralized authority where content } \\
\text { is chosen from a content of materials } \\
\text { designed by the instructor }\end{array}$ \\
\hline Symmetric nature & $\begin{array}{l}\text { Symmetric Learning takes place as an inte- } \\
\text { grated activity }\end{array}$ & $\begin{array}{l}\text { Asymmetric as training takes place } \\
\text { as a separate entity }\end{array}$ \\
\hline Personalization extent & $\begin{array}{l}\text { Personalizes content according to individ- } \\
\text { ual users. }\end{array}$ & $\begin{array}{l}\text { Mass satisfaction is important so } \\
\text { more general vast content }\end{array}$ \\
\hline Adaptability & $\begin{array}{l}\text { Dynamic adaptability where content } \\
\text { changes constantly as per user input, and } \\
\text { self-learning through various experience, } \\
\text { past learning, modules etc. }\end{array}$ & $\begin{array}{l}\text { Static adaptability where Content and } \\
\text { organization retains their original } \\
\text { form authored by instructor/devel- } \\
\text { oper without adhering to external } \\
\text { changes }\end{array}$ \\
\hline
\end{tabular}

\section{Semantic web architecture}

The main concern of ours is the layered architecture of Semantic Web arranged in hierarchy. Each layer is serviceable to the upper layer and acts as client to its underlying layer replicating a vertical pyramid, circular and a towering architecture. As this workflow is sequence driven and arrangement of steps, it represents an abstraction of higher order - alteration in a single layer will be affecting the functionality of other layers. The most notable instance is the International Standards organization (ISO) and the Open Systems Interconnected (OSI) [3]. The newest semantic web architecture as designed by Tim Berners-Lee is explained in the following section.

- Unicode layer and URI provides a simplified means to identify resources like a web site, an image, a document, or a person: basically, any unit with an identity. Unicode, used for computer character representation, is an universal standard encoding system: it represents all language unlike other encoding systems.

- XML layer describes the document content. XML schema on the other hand gives lexical support for authenticated XML documents.

- RDF \& data interchange layer: URI is made use of to target web resources and utilizes graph models to describe relationships among various resources. The schema of RDF is a simplified modelling language that has introduced classes of resources, properties and their interrelationships [4,5].

- SPAQL- query, ontology (OWL), RIF \& RDF-S layer: The backbone of Semantic Web, Ontology, provides semantic executable by the machine \& for better man to application communication - a shareable domain. It's main objective is to provide semantic to generate web meaning, which will help machines decipher the meaning and help in information sharing [5]. The XML format is specified by the RIF (Rule Interchange Format) for rules in compatibility with RDF and OWL at expressive power at an intermediate level according to the RIF Working Group [6]

- Unifying logic layer conveys as an establishment layer for consolidating the two previously mentioned layers into an entire, to direct inquiries and draw in rules over 
information addressed in the RDF alongside ontologies related with it and schemata. Various works in this sector have focused at combining rules with query infused facility, with a combination of rules and grammar.

- Proof layer is for validation of particular statements.

- Trust layer relies upon the data source which can deny undesirable applications or clients admittance to these sources. It's anything but a part of trust and conviction between information sources and units of users.

- UI and application layer acts as an interface the users interacts with so it must satisfy them along with the applications.

- Vertical layers like Crypto is used for encryption purposes and for digital signature. Existing in firth to sixth layer is used to establish web of trust. XML signatures, by applying it to the resource content, it can be identified [7, 8].

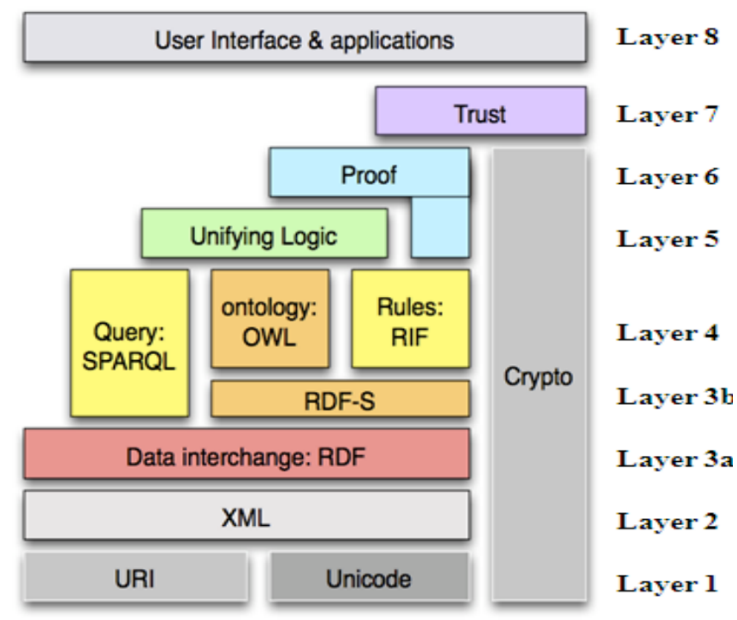

Fig. 1. Diagrammatic Representation Semantic-Web block Architecture

\section{Prerequisites of smart E-learning system}

"E-learning" is in the nick of time instruction incorporated with high speed esteem chains. It is the conveyance of individualized, far reaching, dynamic learning content progressively, supporting the advancement of networks of information, connecting students and professionals with specialists [9].

Prerequisites of delivering consistent, modular, continuous, adaptable and systematic E-Learning system to deliver a distributed, learner-oriented, absolutely personalized dynamic educating system include:

\subsection{Analyzing learner profiles, their requirements and tasks}

After the context is declared, the profile of the learner, that is going to communicate with the mechanism, must be distinguished along with respective work which they are 
going to execute. Uses cases are means to acquire relevant information and relevant requirements in several advanced software development means. These help in the development process to develop a failure or damage proof system considering all aspects and test cases of usability. In [10] Constantine has given few examples of this.

\subsection{Setting the methodology of learning}

It is basically determining the progress and road-map of learning pattern recognizing along with analyzing the requirements and performing parameters and trends of the user, a relevant learning paradigm is defined subjected to alterations. Thus the crucial units of the learning procedure should be properly defined and must be in symphony with the unique features of the user profile.

\subsection{Identifying system parameters}

Entities, a set of states and attributes, are all that a user is going to interact with or use (exams, modules, mails, notification bar etc.). Substances will be utilized for recognizable proof as well as, to expound a glossary with every one of the extraordinary terms that show up in catch stage like approval date, arrangement date and so forth.

\subsection{Identification of extra non-executable units and prerequisites}

These produce features native to the system lie accessibility, availability, reliability etc. that can change few of the executable prerequisites.

\subsection{Exploring the initial flow map}

Navigation of process-flow map is a representation or a layout helping the learner or the user to navigate through the system. This can be thought to be analogous to a hierarchical tree structure in data structure. All the parameters of ease, accessibility, abstraction and simplicity in mind the navigation map is designed and projected to the user as this is the most interacted part by the user and this helps in making the system more usable and acceptable.

\section{$5 \quad$ E-learning and semantic web technologies}

Present search engines are advanced enough but still on search query processing, they still produce unfiltered irrelevant content as well. So this can be solved upon analyzing the profiles and filtering based on that. This can be benefited from the semantic web technologies specially in the E learning sector. This enables semantic web by a collection of units which are suitable to meet important parameters of suitable, efficient and quality E-Learning. Potential sectors of such applications of E Learning applications by Semantic Web are [11] [2]: 


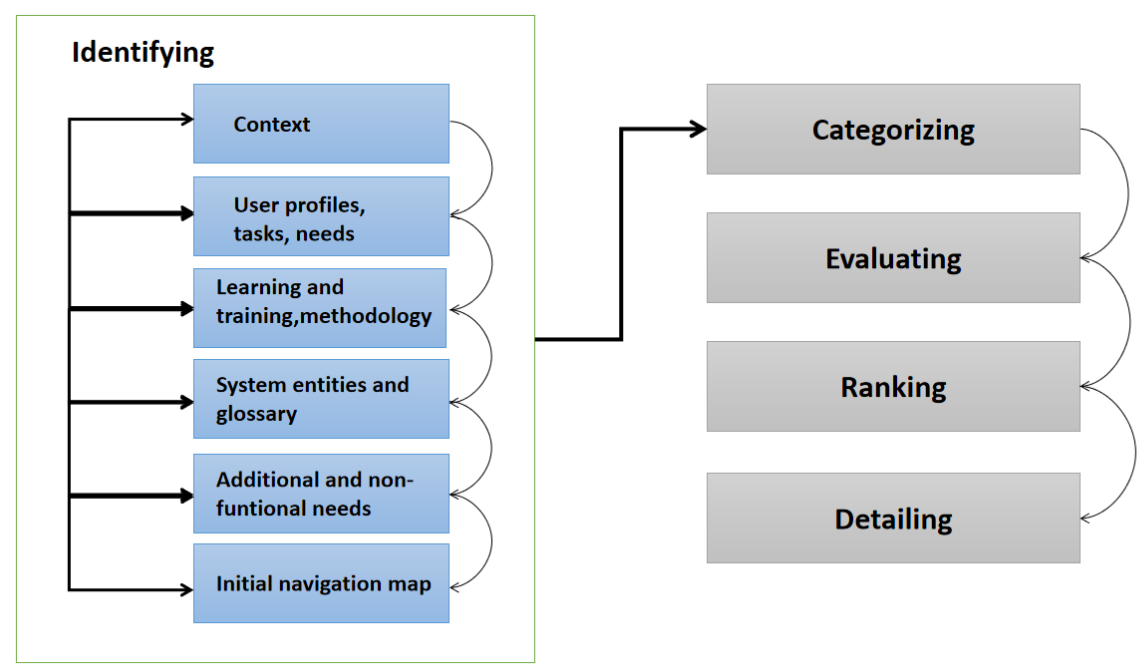

Fig. 2. Learning systems requirement identification process

- Cloud: Distribution of knowledge elements on the world wide web are still at the end lined to an agreed ontology. This direction can be developed for the users through analysis and application of semantic topics that are of mutual interest.

- Inter-activeness: Applications of Semantic Web over mutually immersive languages, more interactive flows, easy and relative flow paths based on profile, helps to increase the user-system interaction.

- Non Linearity: Alternative paths, flows and even based on prior knowledge it should execute the query based on the lexical understanding of relevant learning units.

- Symmetry: Semantic Web offers the service of forming a central integrated system for all executive works under a particular organization including Electronic Learning activities.

- Continuity: Relentless and spontaneous inflow and execution of content based on demand.

- Personnel: Customized and adaptive searches and results and suggestions including educational material and content.

- Distribution: Centralized systems always help in quality maintenance and quality control.

- Dynamic: Distributed nature helps in quality improvement constantly based on previous learning and knowledge.

Learning Objects (LO) as a concept is based on the object-oriented worldview of software engineering and computational sciences [18]. Standard of LOs is the making of educational segments that can be used again on various occasions in various learning settings. Learning Object is a discrete set of educationally stable substance fixated on a learning objective or result proposed to show an engaged idea. It is a major structure block made out of the relative multitude of educationally fundamental parts to involve an independent informative unit. 
This addressed another perspective towards the curriculum on in which substance is separated into discrete pieces (LOs). Educators and students at that point approach the way toward making linkages between pieces to build relevance. These Learning Objects can be conveyed over the internet and can be gotten to by various people all the while, with negligible exertion, decreasing the requirement for teachers to foster their own educational segments. They consider sped up rates and proficiency of informative turn of events and abatement workforce planning time [18].

The major thought behind Learning Objects is that educational originators can fabricate little informative parts that can be reused various occasions in various educational instances [19]. Also, Learning Objects are for the most part perceived to be advanced elements deliverable over the Internet, implying that quite a few groups can access and utilize them all the while.

\subsection{Advantage of learners}

LOs take into consideration making of exceptionally compelling educational encounters for understudies [20] and improving the understudy learning climate [21];

- The decision of which LOs to a mass into an assortment might be taken ahead of time by an informative creator or right now by an understudy [22].

- LOs permit encounters in critical thinking and investigation and coordinated effort with individual students [22];

- LOs permit all-inclusive admittance to electronic educational materials.

- LOs give answers for learning centralized to the learner [20].

\subsection{Instructors benefit with respect to Learning Objects as these}

- are profoundly usable multiple times \& interoperable secluded structure squares or E-learning content dependent on broadly distributed determinations [23];

- advance better joint effort among engineers [20];

- give assets to guidance where there may not in any case be [20];

- can be consolidated in almost limitless manners to develop assortments that may be called exercises, modules, courses or even educational programs.

- consider expanded profitability among coaches and instructors [20];

- offer some benefit as far as setting aside time \& cash in course advancement;

- take into consideration expansions in the multiple usability of substance;

- take into consideration sharing information inside and among sectors;

- connect with workforce in an unique local area of training [21].

\section{Ontologies in the light of smart E-learning environment}

The term Ontologies is famously utilized in the field of computational insight, especially in spaces of coordination and recovery of data, information base administration framework and portrayal of information. The gap in the knowledge 
backgrounds of the learner and the instructor leads to the problem of knowledge retrieval with utmost efficiency and relevance due to use of different terminologies. Ontologies, basically a strong way to solve the said problem by making a distributed conceptualizing and term-related education [2].

It gives a basic job to E-Learning frameworks to officially portray a common importance of a jargon and a bunch of images through a bunch of conceivable planning among images and their implications. In E-learning frameworks, the common perspective issue happens on numerous ontological levels where the depiction of archives can be planned in a few viewpoints.

So, the composition of educational material, it's relation and the structure of the content to avoid isolated learning are the most important issues considered with respect to searches regarding learning material by the learners [13].

Thus, the 3 ontological steps to take considered are situational link, content, and architecture [2].

Context or Situational link ontology deals with the context and intent of the learning domain and includes the interrelationships between their concepts and few basic properties. Ontology should include the transitive property of an element. It can apply relations to capture synonyms and abbreviations.

Contextual issues can be solved as well. Various learning contexts like lectures, examples, training, hands-on etc. help in context-relevant searching.

Structural ontology deals with logical structures of the educational content. In Electronic Learning, for a individual centered paced learning path, it is broken down into modules for more adaptability according to own skill competency. But these should be structured in integrated well to make into an easily accessible and sensible system consisting interleaved and interconnected modules with proper implementations of abstractions and encapsulations. This is normal to foster online-learning frameworks on the internet; subsequently a Web philosophy language ought to be utilized.

Students and Instructors are the main piece of the philosophy based E-learning frameworks and the principal issue to be thought of while building ontologies is the catching and recording the most fundamental utilitarian necessities from students' perspective. The important steps include: identification of aim and the ontology's intention, reusing already available vocabularies, repeating the most valuable terms, class and hierarchy definition, their property definition, definition o the features of these properties, Instance creation [16].

\section{$7 \quad$ Metadata in correlation with E-learning system}

Framework metadata is organized data that portrays assets, which was set up to aid the mission to find, oversee, and use it without being perused, seen or investigated somehow. Metadata is the summation of the information that can be said about the information object at any particular level of aggregation. It will help in the use of multiples usage of a specific thing by distinct communities. This is done via the existence of records regarding metadata, used to portray various plans as per the metadata as per the necessities of each community [13]. Metadata management system 
helps in establishing home EN-Points, used as entry points to the system, to approach teaching, materials etc. It is used to manage the E-Learning system. Ontology is used to structure the detailed demonstration of every management related step and objects the help the educational semantic web. Lytras, Sicily, [14] demonstrate a comparable methodology utilized in displaying objects in learning data frameworks. The said methodology incorporates the determination, establishment, combination and evaluation of learning objects, just as an appraisal of necessities and holes, and educational exercises [15].

\section{Architecture of electronic learning on the lines of web based semantic concept}

To design a smart performing E-Learning system to offer easy access to searched courses, modules etc. Various important things are to me developed and monitored and taken into concern like an information base, and philosophy based data which are the center of the architecture. They are huge for going probably as a vault where ontologies metadata inferring rules, educational resources, course materials and customer profiles are taken care of. The metadata can be situated in the same document or can be preserved outside in an elaborate knowledge unit [14]. Advantages of outside storage or externally stored component include the ease of clearing the separate metadatadescription, stored in the database and thus helps in efficient memory management. Also in terms of re-usability of same materials, there are contradictory approaches by users and authors which sees the possibility of different descriptions according to different concepts [16].

But the most important part will be the user interface GUI with which the user will interact with. Users will interact with it, navigate through it and will get their desired queries. This is the access interface. Semantic search engines provides the API with different methods for conducting queries and knowledge base. The Interference Engine is a shrewd mix of realities that assists with tackling and answer inquiries and is liable for the finish of new real factors through the smart mix of realities as of now have in their insight base. Services include the collection predefined services that are provided to the users like showing details of course, course map etc. In the services section efficient automated customized searching solutions can be included using the implementation of machine learning where the keywords extraction can make the searching results more efficient. The NLP algorithms pre-processes thee searching keywords to find and map results more efficiently. RAKE algorithm is used to extract the keywords with increasing importance beyond a threshold post pre-processing (elimination of stop words, lemmatisation, tokenization etc.). The keywords are then mapped with the topics and course units and from the selected group cosine function helps in the righteous results with most efficiency. 


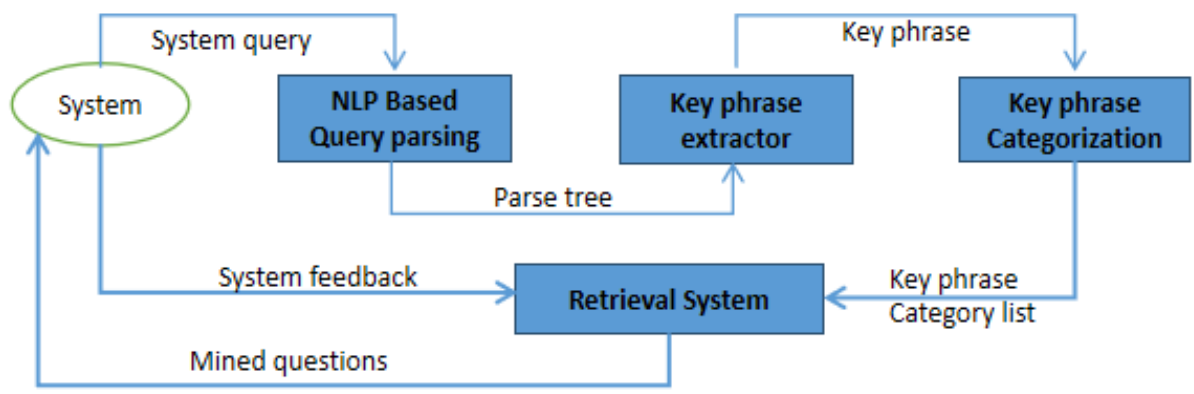

Fig. 3. Proposed Mining Model for Question analysis and information Retrieval for smart relevant searches for smart E-Learning systems.

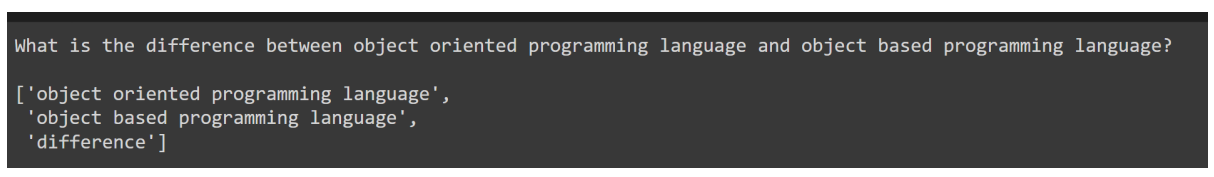

Fig. 4. RAKE-NLTK for key phrase extraction on a sample sentence that extracts the keywords from the sample input statements

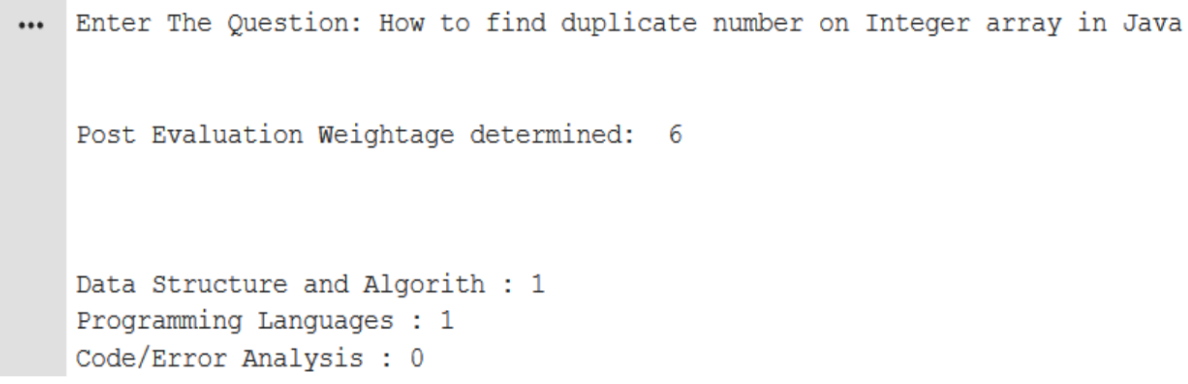

Fig. 5. Class and weightage determination in Sentences to understand and classify outputs

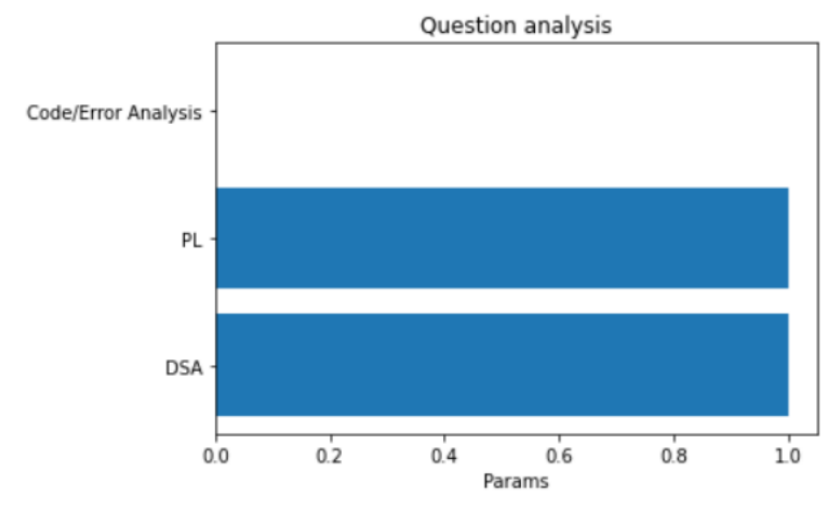

Fig. 6. Key phrase based domain determination 
Customized course and subject combination suggestion can be another feature incorporated in services on analysis of performance and other documents like resumes etc.

The fundamental exercises in electronic learning climate \& give data of the volume, and admittance to instructive components by perusers $\&$ essayists through the inquiry \& perusing.

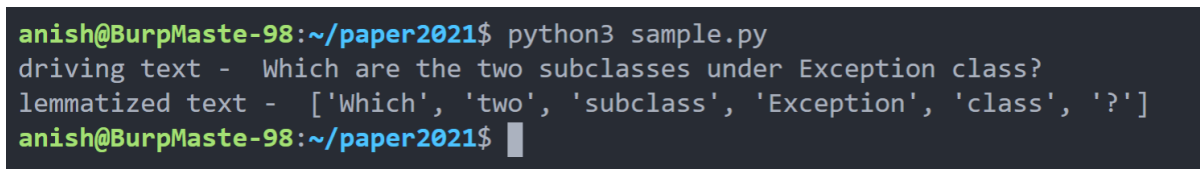

Fig. 7. Lemmatization of texts to find root word

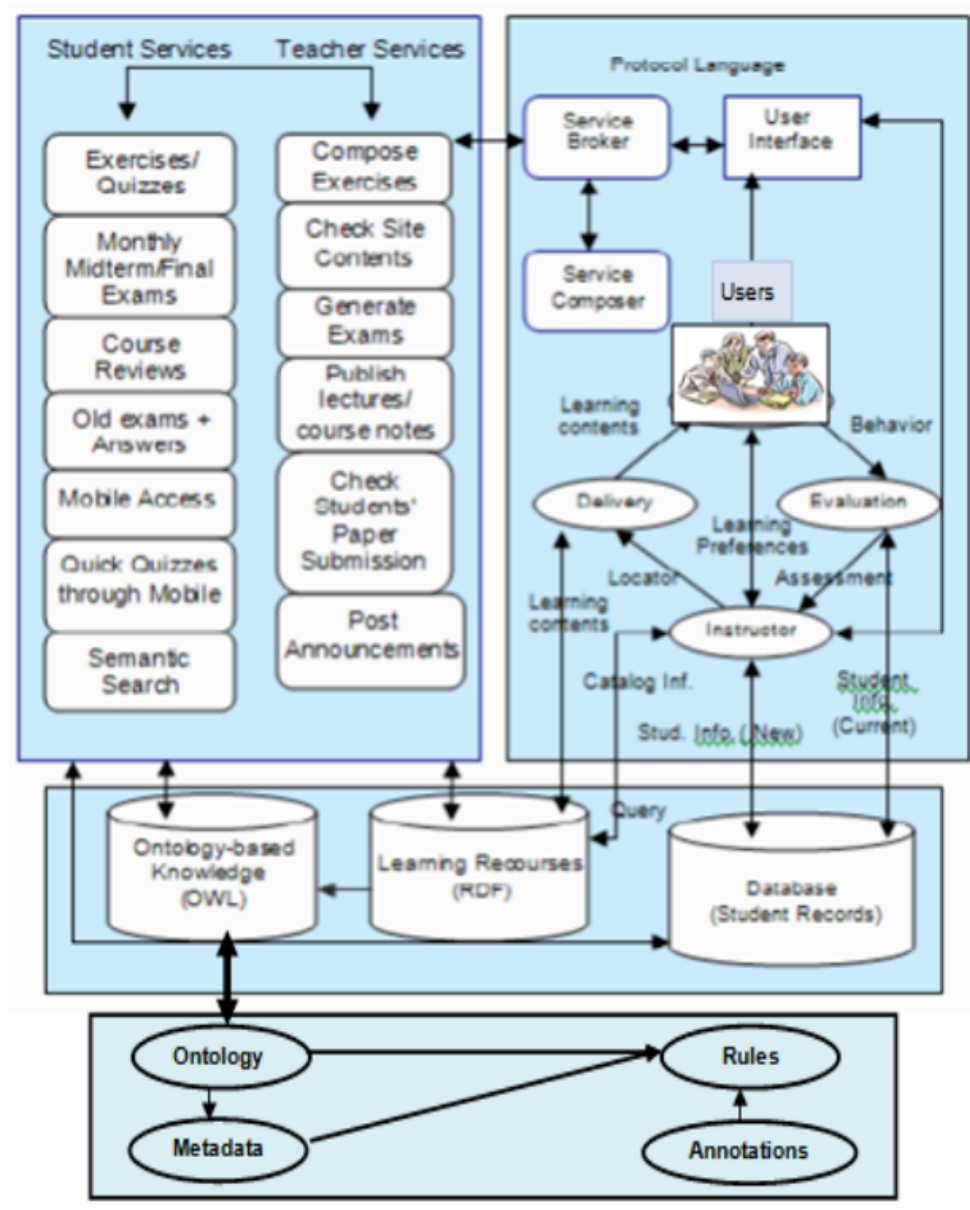

Fig. 8. Electronic learning Model (Conceptual model) on the lines of the Semantic based Web Architecture 


\section{Inference and cessation}

Semantic Web is the arising innovation focusing on electronic data and administrations that would be reasonable and reusable by the two people and machines. One of its essential segments is philosophy, by and large characterized in the form of a portrayal of a common concept of a specific area. It is normal that web related semantics advancements will impact the up and coming age of E-learning frameworks and applications. The thought process of this paper was to feature the benefits semantic web includes that helps the E-Learning framework with a great deal of flexibility, versatility and give a reliable interface to the clients. This reconciliation contemplates the principles guidelines and re-convenience learning objects. portrayals of substance, setting and construction of the educational component and the advantages of giving admittance to the learning material. The creators presented a refreshed E-learning model dependent on the most recent Semantic Web designs. The main aim of this work is to see the advantage of having Semantic web as the backbone of Electronic Learning system but adhering to the main objective of representing a relevant, prompt and easy accessible learning system [17].

\section{Future scope}

Semantic web architecture has shown immense possibilities in making a revolutionary influential outcomes on the prevailing Smart E-Learning systems. The system unit or the architecture can still have innumerable possibilities with the help of machine learning. We have implemented few services like key word extraction and smart search result optimization. There can be other features that can be implemented as well.

\section{Reference}

[1] Berners-Lee. "What the semantic web can represent", 2000 http://www.w3.org/DesignIssues/RDFnot.html.

[2] Dutta, B. Semantic Web Based E-learning, "Semantic Web Based E-learnin", DRTC Conference, ICT for Digital Learning Environment, Bangalore.

[3] Garlan, D. et. al, "An Introduction to Software Architecture. In: Advances in Software Engineering and Knowledge Engineering", World Scientific Publishing Company, NY, 1993.

[4] Buraga, S. and G. Ciobanu., 2002. A RDF- based model for expressing spatio-temporal relation between web sites. In The 3rd International Conference on Information Systems Engineering. IEEE Computer Society. pp: 355. IEEE Computer Society Washington, DC, USA. https://doi.org/10.1109/wise.2002.1181671

[5] Matthews, B, Semantic Web Technologies. JISC Technology and Standards Watch, Joint Information Systems Committee, 2005.

[6] Boley, H. et. al., "RIF Basic Logic Dialect. W3C Working Draft", 2008.

[7] Mark Bartel et al. "XML Signature Syntax and Processing (Second Edition)", 2008.

[8] Russell Cloran et. al, "XML Digital Signature and RDF", 2005 
[9] Drucker P. "Need to Know: Integrating e-Learning with High Velocity Value Chains, A Delphi Group White Paper”, 2000.

[10] Constantine, et. al, "Structure and Style in Use Cases for User Interface Design". 2000.

[11] Stojanovic. L, et. al. E-Learning based on the Semantic Web. IST, 28293, 2001.

[12] Antoniou, Grigoris and Harmelen, Frank van. A semantic web primer, MIT Press, 2004. https://doi.org/10.1017/s0269888905230205

[13] Drucker, P, "Need to Know: Integrating eLearning with High Velocity Value Chains", A Delphi Group White Paper, 2005.

[14] Snae. C et. al," Ontology-Driven E-Learning System Based on Roles and Activities for Thai Learning Environment", Interdisciplinary Journal of Knowledge and Learning Objects, Volume 3, 2007. https://doi.org/10.28945/382

[15] Lytras, M. D.et. al. Modeling the organizational aspects of learning objects in Semantic Web approaches to information systems. Interdisciplinary Journal of Knowledge and Learning Ob-jects, 1, 255-267, 2005. https://doi.org/10.28945/424

[16] Santos et. al. "Design of a Semantic Web-based Brokerage Architecture for the E-learning Domain. A Proposal for a Suitable Ontology", 2005.

[17] Stojanovic et. al. eLearning based on the Semantic Web, 2002.

[18] Freeman, et. al. "Learning objects in microbiology: A new resource". Clinical Laboratory Science, 17(2), 80, 2004.

[19] Wiley et. Al . "Connecting learning objects to instructional design theory: A definition, a metaphor, and a taxonomy". 2000.

[20] Parrish et. al. "The trouble with learning objects. Educational Technology, Research and Development", 52(1), 2004.

[21] EDUCAUSE, Learning objects (2005).

[22] De Salas K. et. al "The Development and Implementation of Learning Objects in a Higher Education Setting", Interdisciplinary Journal of Knowledge and Learning Objects Volume 2, 2006

[23] Clyde et. al. "Digital learning objects. Teacher Librarian", 31(4), 2004.

\section{Authors}

Mr. Subhabrata Sengupta obtained his Bachelor's in Technology degree in the field of Information Technology from Bengal Institute of Technology, India. Then he obtained his Master's degree in the same field from Jadavpur University, India, and is currently pursuing his $\mathrm{PhD}$ in Computer Science majoring in Machine Learning and Information Retrieval from University of Engineering and Management, Kolkata, India. Currently, he is a professor at the Faculty of Information Technology, Institute of Engineering \& Management, Kolkata, India. His specializations are in Machine Learning, Information Retrieval Systems, Smart and adaptive education systems. His current research interests are smart information retrieval systems, student and academic performance analysis, educational data mining and predictive modelling for smart and adaptive education systems with the applications of Machine Learning.

Mr. Anish Banerjee is pursuing his Bacherols in Technology (2017-2021) in Information Technology from Institute of Engineering and Management, Kolkata, India. He worked as a machine Learning Engineer and a Team Lead at Analysed, Hyderabad and as a Machine Learning Developer and consultant at Durbin Technologies, Kolkata, India and is currently placed in Tata Consultancy Services' 
Digital unit. His research interests are in Machine Learning, Deep Learning, Data Analysis and Statistical Modelling of important data. Has has international experience of working in these said fields for companies like MACO, Quinch and FedEX.

Dr. Satyajit Chakrabarti obtained his Bachelor's in Technology degree in the field of Computer Science Engineering from Institute of Engineering and Technology, Kolkata, India (1998-2002). Then he obtained his Master's in Computer Science from The University of British Columbia, Vancouver (2002-2004) and his $\mathrm{PhD}$ in Electronics and Communication Engineering researching in Nanotechnology from National Institute of Technology, Agartala, India (2012-2016). Currently, he is a professor at the Faculty of Computer Science, Institute of Engineering \& Management, Kolkata, India. He was the Teaching and Research Assistant in University of British Columbia, worked as a Developer Analyst and then as a Project Manager in TELUS. He has served as the President of US Chamber of Industry \& Innovation, American Innovation Venture Capital Corporation, American STP Corporation and as the CEO of SAT VENTURES LIMITED. He has always put forward his enthusiasm and support towards productive and innovative ideas and has given his utmost help and co-operation in constructive approaches in the domain of science, research, innovation that builds a better tomorrow.

Article submitted 2021-05-19. Resubmitted 2021-06-26. Final acceptance 2021-06-26. Final version published as submitted by the authors. 